\title{
Plasma in a Pulsed Discharge Environment
}

\author{
Jerome Remy, L. Biennier, and F. Salama
}

\begin{abstract}
The plasma generated in a pulsed slit discharge nozzle is used to form molecular ions in an astrophysically relevant environment. The plasma has been characterized as a glow discharge in the abnormal regime. Laboratory studies help understand the formation processes of polycyclic aromatic hydrocarbon (PAH) ions that are thought to be the source of the ubiquitous unidentified infrared bands.
\end{abstract}

Index Terms-Glow discharge, plasma, polycyclic aromatic hydrocarbon (PAH), pulsed discharge nozzle.

$\mathbf{P}$ OLYCYCLIC aromatic hydrocarbons (PAHs) play an important role in astrophysics. PAHs are found in meteorites and interplanetary dust particles, and they are thought to carry some of the 300 diffuse interstellar bands seen in absorption in diffuse interstellar clouds, as well as the ubiquitous unidentified infrared emission bands. The plasma produced in a pulsed slit discharge nozzle (PDN) source (see Fig. 1) is a perfectly suited environment for generating high column densities of astrochemical species, such as PAH ions, due to the combination of three factors: the significant ionization yield that can be reached in the discharge, a very long slit source, and the low temperature achieved in the postdischarge region, characteristic of the interstellar medium.

The experimental setup has been described in detail elsewhere [1]. The PDN source consists of a supersonic slit free jet mounted in a vacuum chamber and two knife-edge electrodes that create a discharge in the stream of the planar expansion. The plasma is thus generated after the gas, which is composed of highly diluted PAHs in Ar or Ne, expands. Two negatively biased jaws forming the cathode are mounted on each side of the slit and insulated from the PDN assembly (anode). The applied voltage ( -400 to $-600 \mathrm{~V}$ through $1-\mathrm{k} \Omega$ ballast resistors) ensures that electrons flow against the supersonic stream and that the discharge remains confined, as well as uniform within the 10-cm long slit. At typical backing pressure of 760 torr, detailed flow simulations show that the average pressure within the thin plasma region is of the order of 80 torr [2].

Note that all photographs are static images captured with an ordinary digital camcorder (no filter), which shows how easily the PDN plasma expansion can be visualized.

Manuscript received July 3, 2004; revised December 1, 2004. This work was supported by the NASA APRA Program and by the Center for Plasma Physics and Radiation Technology (CPS).

J. Remy is with the Applied Physics Department, Eindhoven University of Technology, 5600 MB Eindhoven, The Netherlands.

L. Biennier is with the Laboratoire de Physique des Atomes, Lasers, Molécules et Surfaces (PALMS), Université de Rennes, 35042 Rennes Cedex, France.

F. Salama is with the NASA Ames Research Center, Moffett Field, CA $94035-$ 1000 USA.

Digital Object Identifier 10.1109/TPS.2005.845937

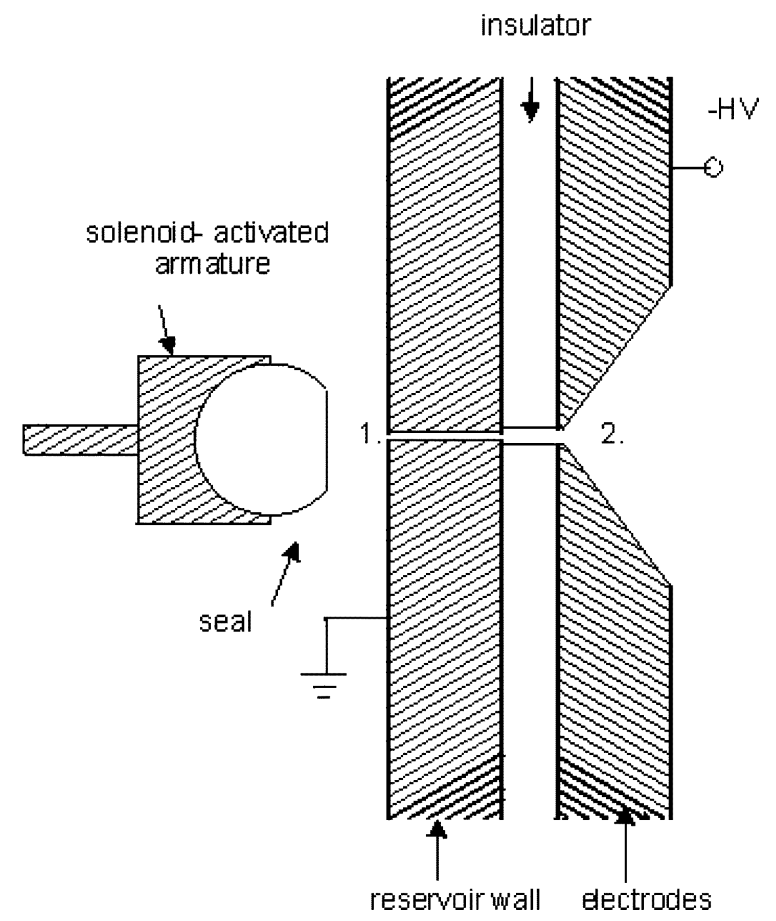

Fig. 1. Cross section of the PDN source. Solenoid-activated armature injects a 1.2-ms-pulse of gas into the plasma source. (1) points to the $200 \mu \mathrm{m} \times 10 \mathrm{~cm}$ slit; (2) $400-\mu \mathrm{m}$ gap between the two jaws forming the cathode. Note that the distance between anode and cathode is $1.5 \mathrm{~mm}$.

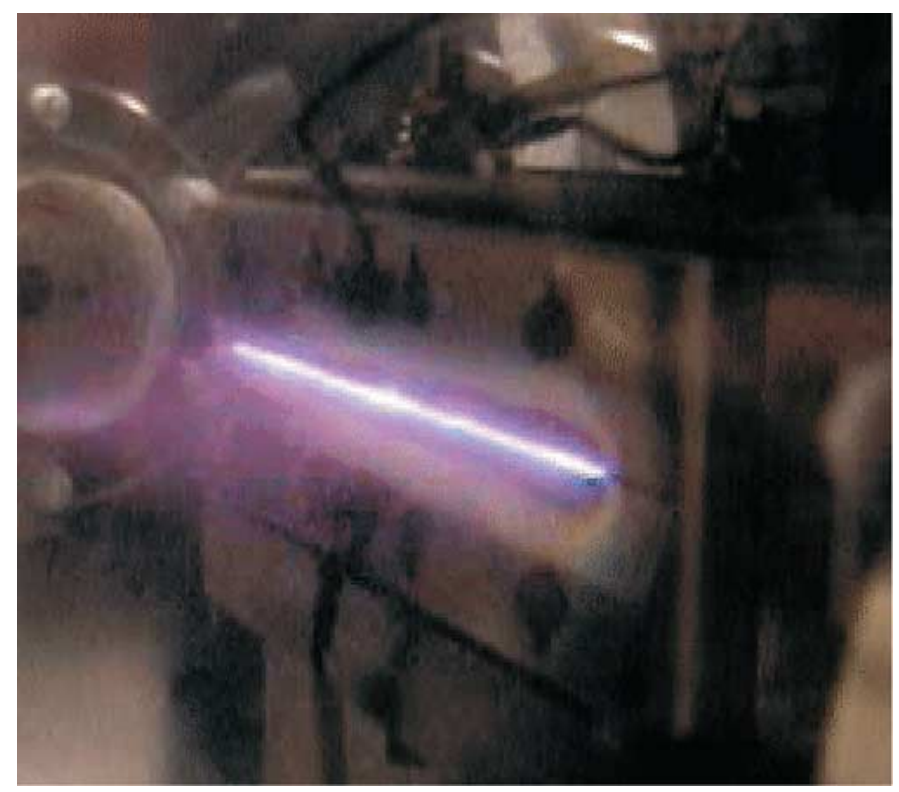

Fig. 2. Picture of an Ar plasma generated in the PDN source. Plasma is generated uniformly along the slit, allowing for high column densities of PAH ions. Variation of the $\mathrm{Ar}_{\mathrm{m}}$ and $\mathrm{Ar}^{+}$densities in the vicinity of the cathode helps explain the brightness difference that is seen between the slit and the expansion itself. 

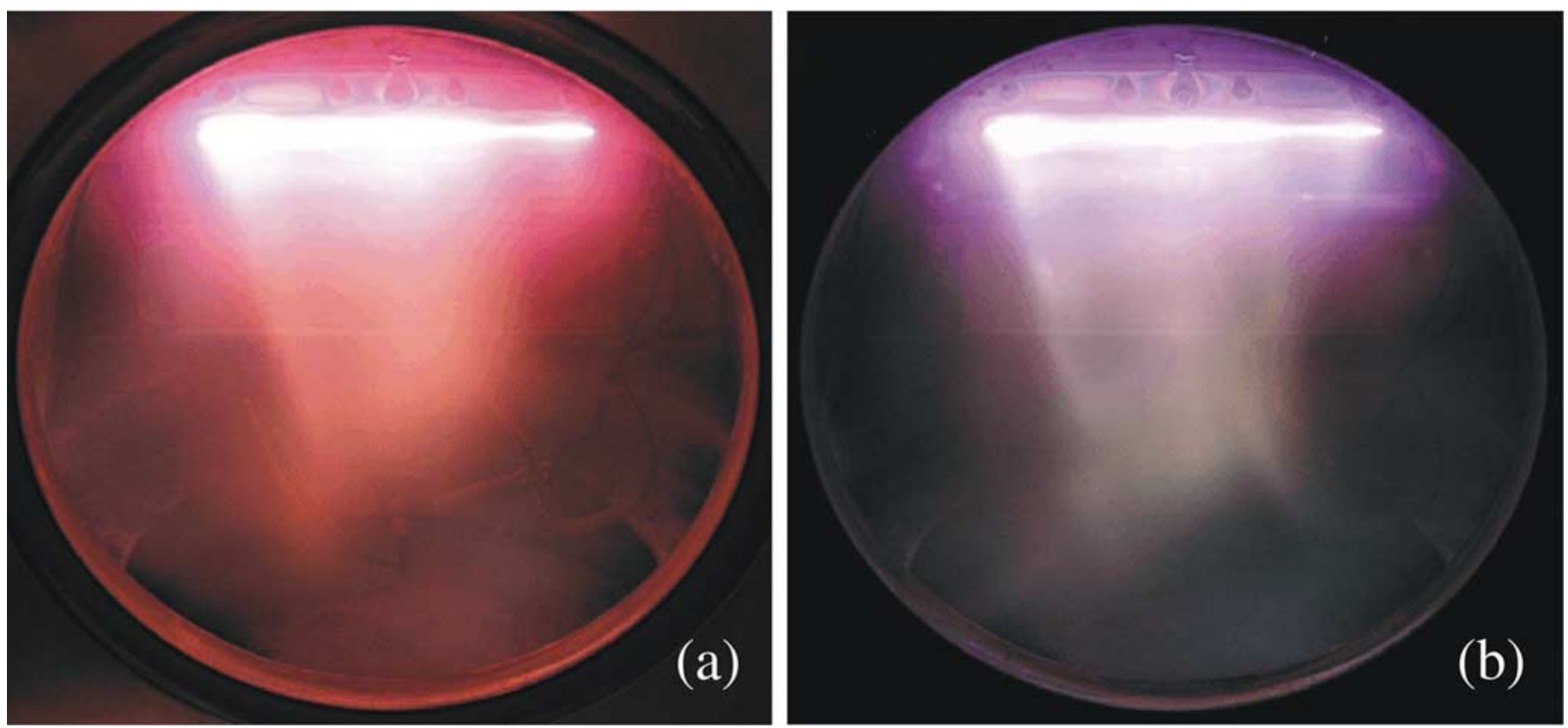

Fig. 3. Pictures of the (a) Ne and (b) Ar plasma expanding in the vacuum chamber at a residual pressure of 150 mtorr. Plasmas are very well confined within the cathode jaws, highlighting the presence of a high background gas density near the cathode. At high voltages, soot deposit on the cathode's jaws after plasma ignition and disturb the homogeneity of the light emission.

The discharge generated in the PDN has been identified as an abnormal glow discharge, characterized by the sole presence of a negative glow and sheaths at the source's boundaries [3]. The typical light emitted by the plasma source, with argon as the carrier gas, is shown in Fig. 2. As expected, a blue-purplish light emerges from the slit in the expansion plane, confirming the absence of a positive column in the glow discharge. The light originates from photons emitted by radiative relaxation of the excited metastable $\mathrm{Ar}_{\mathrm{m}}$ atoms and from excited $\mathrm{Ar}^{+}$ions. Electrons are accelerated in the cathode's sheath and gain enough energy to excite high energy levels of $\mathrm{Ar}_{\mathrm{m}}$ and $\mathrm{Ar}^{+}$near the cathode jaws [4]. Fig. 2 not only displays a very bright active plasma, confirming our previous statement, but also shows that light is uniformly generated along the slit.

Shortly after plasma ignition, accumulation of PAH soot can be noticed on the electrodes for high voltages, leading to a loss of the emission uniformity at the slit's exit, as seen in Figs. 3(a) and (b). This indicates that chemistry is taking place in the plasma, despite the small size of the active region. More importantly, Fig. 3 also shows that the plasma is strictly confined in the vicinity of the slit, indicating the presence of a very high background gas density in that region. The weaker brightness of the plasma expansion in the vacuum chamber points to a chemically less active zone (with lower $\mathrm{Ar}_{\mathrm{m}}$ and $\mathrm{Ar}^{+}$densities), concurring with the thermodynamic properties of a supersonic expansion.
Recent numerical simulations of the source operation [4] have confirmed the empirical approach used in this work.

\section{ACKNOWLEDGMENT}

The authors wish to thank R. Walker of NASA Ames for his invaluable technical support, as well as Prof. G. M. W. Kroesen and Dr. W. W. Stoffels from the Technische Universiteit Eindhoven for their useful comments and discussions. This work was performed at NASA Ames Research Center, Moffett Field, CA.

\section{REFERENCES}

[1] L. Biennier, F. Salama, L. J. Allamandola, and J. J. Scherer, "Pulsed discharge nozzle cavity ring-down spectroscopy of cold polycyclic aromatic hydrocarbon ions," J. Chem. Phys., vol. 118, no. 17, pp. 7863-7872, 2003.

[2] L. Biennier, A. Benidar, and F. Salama, "Flow dynamics of a pulsed planar expansion,", submitted for publication.

[3] J. Remy, L. Biennier, and F. Salama, "Plasma structure in a pulsed discharge environment," Plasma Sources Sci. Technol., vol. 12, pp. 295-301, 2003.

[4] B. H. P. Broks, W. J. M. Brok, J. Remy, J. J. A. M. van der Mullen, A. Benidar, L. Biennier, and F. Salama, "Numerical investigation of the discharge characteristics of the pulsed discharge nozzle," Phys. Rev. E, Stat. Phys. Plasmas Fluids Relat. Interdiscip. Top., vol. 71, 2005. 